\title{
Incorporating Game-theoretic Rough Sets in Web-based Medical Decision Support Systems
}

\author{
JingTao Yao and Nouman Azam \\ Department of Computer Science, University of Regina, Regina, Saskatchewan, Canada S4S 0A2 \\ Email $\{$ jtyao,azam200n\}@cs.uregina.ca
}

\begin{abstract}
Web-based support systems (WSS) assist human activities with the modern Web technology. An important branch of WSS is Web-based decision support systems that provide intelligent support for decision making tasks. We focus on decision making in Web-based medical decision support systems (WMDSS) that can provide support for making diagnosis and treatment decisions. The use of game-theoretic rough set (GTRS) component in WMDSS is explored and investigated for this purpose. The GTRS is a recent development in rough sets that takes advantages from data analysis capabilities of rough sets complimented with decision analysis abilities of game theory. The GTRS may be used to obtain rough sets based threeway or ternary decisions by determining a pair of threshold values. Demonstrative example suggests that the GTRS may be considered as an alternative decision making model and component in building WMDSS for providing decision support.
\end{abstract}

\section{INTRODUCTION}

The study of Web-based support systems (WSS) aims at developing and transforming existing systems in different domains to assist in various human activities over the Web [5], [11], [13]. The WSS take advantages from developments and opportunities provided by the Web such as advances in information processing, collecting, storing, searching, retrieving, sharing and using over the Web [15]. We focus on decision making in Web-based medical decision support systems (WMDSS) that aid the practitioners in making treatment and diagnosis decisions [4].

Uncertainty is a critical issue that affects medical decision making [8]. The factors, such as difficulties in exactly expressing an observation, effectiveness of treatment and precision of medical tests, are some issues that makes the decision making a challenging job. The presence of such factors demands for an effective decision making component for WMDSS.

A ternary decision making approach is commonly employed in medical decision making to reduce the effects of uncertainty. A known model in this regard was proposed by Pauker and Kassier for making treatment decisions [10]. A pair of testing and test-treatment thresholds were used to define and obtain the decisions of treatment, no treatment and delay treatment. The delay or deferment decision option assumes that further information may evolve which will make the decision making more evident and certain. Recently, Yao established the theory of three-way decisions [18]. The theory helps to explain how to induce and interpret three-way decision rules obtained

Acknowledgements: This work was partially supported by a Discovery Grant from NSERC Canada. with the set related theories such as fuzzy sets, rough sets, shadowed sets and interval sets. This potentially opened new research avenues for exploring existing methods and models based on set related theories in building interesting WMDSS with ternary decision making capabilities. The game-theoretic rough sets (GTRS) provide one such method that can be used to obtain three-way decisions. The importance of GTRS is its ability to automatically learn the threshold parameters (used to induce decision rules) by realizing the optimization of one or more characteristics of decision making in a soft computing paradigm.

We propose a WMDSS that incorporates a GTRS component for evaluating and providing decision recommendations. The GTRS provide a threshold configuration mechanism that are used to define and control the size of positive, negative and boundary regions in the probabilistic rough set model [1], [7]. The three regions may be interpreted as regions of acceptance, rejection and deferment. A demonstrative example suggests that the inclusion of GTRS component in WMDSS can be useful for obtaining ternary decision recommendations.

\section{Decision Making With Game-theoretic ROUGH SETS}

The GTRS model determines the thresholds that control the size of positive, negative and boundary regions for a given concept in a probabilistic rough set setting [3], [7]. A main result of probabilistic rough sets is that the rules for determining the three regions are defined by,

$$
\begin{array}{ll}
\text { Positive: } & \text { if } P(C \mid[x]) \geq \alpha, \\
\text { Negative: } & \text { if } P(C \mid[x]) \leq \beta, \text { and } \\
\text { Boundary: } & \text { if } \beta<P(C \mid[x])<\alpha .
\end{array}
$$

where $P(C[x])$ is the conditional probability of an object $x$ to be in $C$ provided that the object is in equivalence class given by $[x]$ and $0 \leq \beta<\alpha \leq 1$. The positive, negative and boundary regions are sometimes interpreted as regions of acceptance, rejection and deferment, respectively [18]. The division between these three regions is based on the probabilistic thresholds $(\alpha, \beta)$ [16]. How to determine and interpret the thresholds are among the fundamental issues in probabilistic rough sets [17]. There are at least three approaches to determine the thresholds known as decision-theoretic rough sets [16], game-theoretic rough sets (GTRS) [3], [7] and 
TABLE I: A two-player GTRS based game for calculation thresholds

\begin{tabular}{ccccc}
\hline & & \multicolumn{3}{c}{$C^{\prime \prime}$} \\
\cline { 2 - 4 } & & $t_{1}=h_{1}(\alpha, \beta)$ & $t_{2}=h_{2}(\alpha, \beta)$ & $\cdots$ \\
\hline \multirow{3}{*}{$C^{\prime}$} & $s_{1}=f_{1}(\alpha, \beta)$ & $\left(\alpha_{1}, \beta_{1}\right) \Longrightarrow\left(c_{1}^{\prime}, c_{1}^{\prime \prime}\right)$ & $\left(\alpha_{2}, \beta_{2}\right) \Longrightarrow\left(c_{2}^{\prime}, c_{2}^{\prime \prime}\right)$ & $\cdots$ \\
\cline { 2 - 5 } & $s_{2}=f_{2}(\alpha, \beta)$ & $\left(\alpha_{3}, \beta_{3}\right) \Longrightarrow\left(c_{3}^{\prime}, c_{3}^{\prime \prime}\right)$ & $\left(\alpha_{4}, \beta_{4}\right) \Longrightarrow\left(c_{4}^{\prime}, c_{4}^{\prime \prime}\right)$ & $\cdots$ \\
\cline { 2 - 5 } & & $\cdots$ & $\cdots$ & $\cdots$ \\
\hline
\end{tabular}

information theoretic rough sets [6], respectively.

The GTRS based threshold determination is obtained in a formulated game [2]. A game may be defined as a tuple $\{P, S, u\}$, where [9]:

- $P$ is a finite set of $n$ players, indexed by $i$,

- $S=S_{1} \times \ldots \times S_{n}$, where $S_{i}$ is a finite set of strategies available to player $i$. Each vector $s=\left(s_{1}, s_{2}, \ldots, s_{n}\right) \in S$ is a strategy profile where player $i$ selects strategy $s_{i}$.

- $u=\left(u_{1}, \ldots, u_{n}\right)$ where $u_{i}: S \longmapsto \Re$ is a real-valued utility or payoff function for player $i$.

Considering $s_{-i}=\left(s_{1}, s_{2}, \ldots, s_{i-1}, s_{i+1}, \ldots, s_{n}\right)$ be a strategy profile without $i$ th player strategy, we may write $s=$ $\left(s_{1}, s_{2}, \ldots, s_{n}\right)=\left(s_{i}, s_{-i}\right)$. This means all the players expect $i$ are committed to play $s_{-i}$ while player $i$ choose $s_{i}$. The strategy profile $\left(s_{1}, s_{2}, \ldots, s_{n}\right)$ is a Nash equilibruim [9], if $s_{i}$ is a best response to $s_{-i}$ for all $i$, that is,

$$
u_{i}\left(s_{i}, s_{-i}\right) \geq u_{i}\left(s_{i}^{\prime}, s_{-i}\right), \quad \text { where }\left(s_{i}^{\prime} \in S_{i} \wedge s_{i}^{\prime} \neq s_{i}\right)
$$

This means that Nash equilibrium is a strategy profile in which none of the players can be benefited by changing their respective strategies, given the other players chosen action.

In a GTRS based game, the players are considered in the form of multiple criteria where each criterion represents a particular aspect of rough set based classification. For instance, the criteria such as accuracy and generality of the rough set model can be considered as possible players. The strategies are formulated in terms of changes in probabilistic thresholds [1]. The payoff functions represents possible gains, benefits or performance levels achieved by considering different modification of threshold levels.

Table I represents a general form of a two-player game formulated with the GTRS. The players are denoted as $C^{\prime}$ and $C^{\prime \prime}$ representing different criteria. The strategies are shown as functions of $(\alpha, \beta)$ that can configure the thresholds in some order. Each cell of the table represents a strategy profile e.g. $\left\langle s_{1}, t_{1}>\right.$ that leads to a threshold pair $\left(\alpha_{1}, \beta_{1}\right)$. The payoff functions or utilities evaluated with respective criteria corresponding to a particular set of threshold pair, say $\left(\alpha_{1}, \beta_{1}\right)$, are represented as $\left(c_{1}^{\prime}, c_{1}^{\prime \prime}\right)$. The solution concept of Nash equilibrium can be used to determine the game outcome and the associated threshold pair. The determined thresholds can then be used to induce three-way decisions in the probabilistic rough sets framework.

\section{Conceptual Model of Web-Based Medical DECISION SUPPORT SYSTEMS}

The WMDSS contain various components with functionalities ranging from supporting end user activities and interaction through interface to maintaining and manipulating the knowledge within the system. Fig. 1 presents a conceptual model of Web-based medical decision support systems. The detailed architecture may be found in references [12], [14]. The model may be understood as comprising of interface, management and data layers.

\begin{tabular}{|c|}
\hline $\begin{array}{c}\text { Interface Layer } \\
\text { Web, Internet, Presentation }\end{array}$ \\
\hline $\begin{array}{c}\text { Mans, Knowledge Managment, GTRS, } \\
\text { Information Retrieval, Control facilities }\end{array}$ \\
\hline Data Layer \\
Patient Database, Thresholds Database, \\
Three-way Rules Database
\end{tabular}

Fig. 1: Conceptual Model of WMDSS

\section{A. Interface Layer}

The web and internet makes up the interface layer. The clients interact with the system that is designed on the server side through an interface that is supported by the Web and internet. The interface is presented to the clients with the help of Web browsers. The interface is not only used by the users to input any relevant information that the system may require but also is responsible for providing services and functionalities like searching, storing or obtaining decision support corresponding to a patient. A carefully designed web interface is very critical for the success of the whole system. It has to be clear, complete, consistent and easy to interpret. In addition it should also provide user guidance and auto correction facilities.

\section{B. Management Layer}

This layer serve as middleware of the three layer model. The information from the top or bottom layer are processed at this layer before being presented to an intended upper or lower 
layer. Intelligent techniques to analyze data such as logic, inference and reasoning play a key role at this layer.

We briefly describe the components that may be required for an effective implementation at this layer. 1) Database Management System (DBMS): The DBMS binds the patient database and the GTRS component. The data made available from the database will be used by the GTRS to learn rules for ternary decision making. 2) Knowledge Management: The knowledge management provides access to the knowledge base which contains the rules database and thresholds database. The three-way rules and the associated threshold values are learned from the data with the help of the GTRS component. 3) GTRS Component: It utilizes a game-theoretic environment in analyzing rough sets to acquire knowledge in the form of three-way decision rules. The rules are accompanied by the knowledge related to threshold levels for acceptance, rejection and deferment of rules. 4) Information Retrieval: This component is responsible for handling user queries related to patient's data, information from knowledge base and other related tasks. 5) Control Facilities: Control facilities include security checks such as access rights and permissions. As the patients information is highly confidential, the web functionality introduces new challenges for securing the data.

\section{Data Layer}

This layer contains data necessary for the operation of the system. The bulk of this data appears in the form of patient database. The database is populated with data by conducting series of questions and trails performed on patients. From decision making perspective, the symptoms corresponding to a disease makeup the most important part of this database.

This layer also contains the knowledge base component which contains information about three-way rules in the rules database. These rules are learned from the data using the GTRS component and are utilized to make decisions of acceptance, rejection and deferment. The rules are accompanied by the threshold levels in the threshold database. This database contains the associated threshold levels or confidence levels in making ternary decisions.

\section{INCORPORATING GTRS IN WEB-BASED MEDICAL DECISION SUPPORT SYSTEMS}

To illustrate how a GTRS component can assist in decision making in WMDSS, we consider a case for diagnosing a single disease. The system can recommends diagnosis decisions based on some physiological properties recorded in the form symptoms or medical tests corresponding to a disease. Table II presents information about a disease. The rows of the table represent the information of each patient and the columns are different characteristics shown by patients. The last columns is the diagnosis decision made in the past corresponding to a patient.

Let $X_{i}$ represents an equivalence class which is the set of patients having the same description, i.e. patients with same
TABLE II: A partial information table containing patients data

\begin{tabular}{|c|c|c|c|c|}
\hline Patient & $S_{1}$ & $S_{2}$ & $S_{3}$ & Decision \\
\hline$P_{1}$ & 1 & 1 & 1 & Yes \\
$P_{2}, P_{3}$ & 1 & 1 & 0 & Yes \\
$P_{4}, P_{5}, P_{6}, P_{7}$ & 1 & 0 & 1 & Yes \\
$P_{8}$ & 1 & 0 & 1 & No \\
$P_{9}, P_{10}, P_{11}$ & 0 & 1 & 1 & Yes \\
$P_{12}$ & 0 & 1 & 1 & No \\
$P_{13}, P_{14}$ & 0 & 1 & 0 & Yes \\
$P_{15}, P_{16}$ & 0 & 1 & 0 & No \\
$P_{17}, P_{18}$ & 0 & 0 & 1 & No \\
$P_{19}$ & 0 & 0 & 1 & Yes \\
$P_{20}, P_{21}, P_{22}, P_{23}$ & 1 & 0 & 0 & No \\
$P_{24}$ & 1 & 0 & 0 & Yes \\
$P_{25}, P_{26}, P_{27}$ & 0 & 0 & 0 & No \\
\hline
\end{tabular}

values for $S_{1}, S_{2}$ and $S_{3}$. The following equivalence classes may be formed based on Table II.

$$
\begin{array}{ll}
X_{1}=\left\{P_{1}\right\}, & X_{2}=\left\{P_{2}, P_{3}\right\}, \\
X_{3}=\left\{P_{4}, P_{5}, P_{6}, P_{7}, P_{8}\right\}, & X_{4}=\left\{P_{9}, P_{10}, P_{11}, P_{12}\right\}, \\
X_{5}=\left\{P_{13}, P_{14}, P_{15}, P_{16}\right\}, & X_{6}=\left\{P_{17}, P_{18}, P_{19}\right\}, \\
X_{7}=\left\{P_{20}, P_{21}, P_{22}, P_{23}, P_{24}\right\}, & X_{8}=\left\{P_{25}, P_{26}, P_{27}\right\} .
\end{array}
$$

The conditional probability for positively diagnosing a disease based on $X_{i}$ is given by,

$$
P\left(\text { Decision }=\text { Yes } \mid X_{i}\right)=\frac{\mid \text { Decision }=Y \text { es } \bigcap X_{i} \mid}{\left|X_{i}\right|} .
$$

The conditional probabilities, based on Equation (3) of equivalence classes $X_{1}, \ldots, X_{8}$, determined from Table II are $1.0,1.0,0.8,0.75,0.5,0.33,0.2$ and 0.0 , respectively.

Let us consider the properties of accuracy and generality of diagnosing decisions. The accuracy refers to the number of correct diagnosis and the generality refers to the number of patients for whom diagnosing decisions can be made. It may not be possible based on the available evidence to obtain accurate diagnosis results for all patients. In general, a decision model with high accuracy may have lesser generality while a model with high generality may have a lower accuracy [3], [6].

For a group containing both positive and negative regions we may define accuracy and generality measures as [1], [3],

$$
\begin{aligned}
& \text { Acc. }(\alpha, \beta)=\frac{\text { Correctly classified objects by } \operatorname{POS}_{(\alpha, \beta)} \text { and } \mathrm{NEG}_{(\alpha, \beta)}}{\text { Total classified objects by } \operatorname{POS}_{(\alpha, \beta)} \text { and } \mathrm{NEG}_{(\alpha, \beta)}}, \\
& \text { Gen. }(\alpha, \beta)=\frac{\text { Total classified objects by } \operatorname{POS}_{(\alpha, \beta)} \text { and } \mathrm{NEG}_{(\alpha, \beta)}}{\text { Number of objects in } U},
\end{aligned}
$$

where $U$ is the total number of objects. The two measure may be calculated based on information in Table II (refer to [3] for detailed calculations). The configuration of thresholds $(\alpha, \beta)$ to increase one property may affect the other. The GTRS based approach outlined in section II may be utilized to implement a game between the two properties to determine effective thresholds.

Let us consider a game having these two measures as play- 
ers. Each player may choose from three possible strategies, namely, $s_{1}=\alpha_{\downarrow}=$ decrease $\alpha, s_{2}=\beta_{\uparrow}=$ increase $\beta$, and $s_{3}=\alpha_{\downarrow} \beta_{\uparrow}=$ decrease $\alpha$ and increase $\beta$. In this example, we consider an increase or decrease of $25 \%$ and initial values of $(\alpha, \beta)=(1,0)$. This means that strategy $s_{1}$ can be interpreted as $25 \%$ decrease in $\alpha$ which leads to $\alpha=0.75$. In order to determine a threshold pair corresponding to a strategy profile we differentiate between two possible cases. During the game if only a single player suggests a change in a threshold value, the value will be determined as an increase or decrease suggested by that player. Alternatively, if both the players suggest a change, the value will be decided as the sum of the two changes. Based on these two cases, a threshold pair corresponding to a strategy $\left(s_{1}, s_{2}\right)=\left(\alpha_{\downarrow}, \beta_{\uparrow}\right)$ will be determined as $(\alpha, \beta)=(0.75,0.25)$.

TABLE III: Payoff table for the example game

\begin{tabular}{ccccc}
\hline & & \multicolumn{4}{c}{ Gen. } \\
\cline { 2 - 5 } & & $\alpha_{\downarrow}$ & $\beta_{\uparrow}$ & $\alpha_{\downarrow} \beta_{\uparrow}$ \\
\hline \multirow{3}{*}{ Acc. } & $\alpha_{\downarrow}$ & $0.79,0.70$ & $0.85,0.74$ & $0.79,0.88$ \\
\cline { 2 - 5 } & $\beta_{\uparrow}$ & $0.85,0.74$ & $0.90,0.41$ & $\mathbf{0 . 8 3 , 0 . 8 5}$ \\
\cline { 2 - 5 } & $\alpha_{\downarrow} \beta_{\uparrow}$ & $0.79,0.88$ & $0.83,0.85$ & $0.77,1.0$ \\
\hline
\end{tabular}

Table III represents the payoff table corresponding to the game. The cell containing bold values, i.e. (0.83,0.85) with corresponding strategy pair $\left(\beta_{\uparrow}, \alpha_{\downarrow} \beta_{\uparrow}\right)$ is a solution of the game defined by the Nash equilibrium. This means that none of the players can achieve a higher payoff, given the other player's chosen action. The threshold pair corresponding to this strategy profile is given by $(\alpha, \beta)=(0.75,0.5)$. The calculated thresholds with the GTRS can be used to generate ternary decisions as outlined in Equation (1).

We may interpret the GTRS results from a medical practitioner perspective. Considering a patient $x$ with its equivalence class $[x]$ and respective conditional probability $P(C \mid[x])$. We can make $83 \%$ correct diagnosis decisions if the level of confidence for accepting $x$ to have a disease is greater than or equal to 0.75 and the level of confidence for rejecting $x$ to have a disease is lesser than or equal to 0.5 . However, these decisions are applicable to $85 \%$ of the patients.

The GTRS based decisions may be further improved by considering the nature or type of a particular disease. For instance, in case of cancer diagnosing, the accuracy is very critical. One may formulate game strategies that can provide higher payoffs to the player accuracy. The GTRS provided an interesting alternative for building WMDSS that can provide decision support in the form of ternary decision recommendations.

\section{CONCLUSION}

We examine Web-based medical decision support systems that incorporates a game-theoretic rough set component for making three-way or ternary decisions. The proposed approach adds a third option of deferment or delaying a certain decision.
This option provides some flexibility in situations where the available evidence is not sufficient to reach or make a certain decision in the form of acceptance or rejection. The use of GTRS component in the WMDSS is explained to obtain threeway decisions in the probabilistic rough set setting. Particularly, the GTRS component obtains the threshold parameters that defines the three probabilistic regions and the implied three-way decisions. The demonstrative example advocates for the possibility of considering GTRS in building WMDSS for providing decision support in the form of ternary decisions.

In future, we wish to explore the capabilities of GTRS on medical data and compare the results with some of the contemporary approaches.

\section{REFERENCES}

[1] N. Azam and J. T. Yao, "Multiple criteria decision analysis with gametheoretic rough sets," in Proceedings of 7 th International Conference on Rough Sets and knowledge Techonology (RSKT'12), Lecture Notes in Computer Science 7414, 2012, pp. 399-408.

[2] N. Azam and J. T. Yao, "On semantic issues in game-theoretic rough sets," in Proceedings of Joint IFSA World Congress and NAFIPS Annual Meeting (IFSA/NAFIPS), 2013, pp. 1303-1308.

[3] N. Azam and J. T. Yao, "Analyzing uncertainties of probabilistic rough set regions with game-theoretic rough sets," International journal of approximate reasoning, http://dx.doi.org/10.1016/j.ijar.2013.03.015, 2013.

[4] E. S. Berner and J. J. McGowan, "Use of diagnostic decision support systems in medical education," Methods of Information in Medicine, vol. 49 , no. 4, pp. 412-417, 2012.

[5] H. K. Bhargava, D. J. Power, and D. Sun, "Progress in web-based decision support technologies," Decision Support Systems, vol. 43, no. 4, pp. 1083-1095, 2007.

[6] X. F. Deng and Y. Y. Yao, "An information-theoretic interpretation of thresholds in probabilistic rough sets," in Proceedings of Rough Sets and Current Trends in Computing (RSCTC'12), Lecture Notes in Computer Science 7413, 2012, pp. 232-241.

[7] J. P. Herbert and J. T. Yao, "Game-theoretic rough sets," Fundamenta Informaticae, vol. 108, no. 3-4, pp. 267-286, 2011.

[8] B. G. Koerkamp, M. C. Weinstein, T. Stijnen, M. H. Heijenbrok-Kal, and M. M. Hunink, "Uncertainty and patient heterogeneity in medical decision models," Medical Decision Making, vol. 30, no. 2, pp. 194-205, 2010.

[9] K. Leyton-Brown and Y. Shoham, Essentials of Game Theory: A Concise Multidisciplinary Introduction. Morgan \& Claypool Publishers, 2008.

[10] S. G. Pauker and J. P. Kassirer, "The threshold approach to clinical decision making," The New England Journal of Medicine, vol. 302, no. 20 , pp. $1109-1117,1980$.

[11] J. T. Yao, "On web-based support systems," in Proceedings of the 2nd Indian International Conference on Artificial Intelligence, (IICIA'05), 2005, pp. 2589-2600.

[12] J. T. Yao and J. P. Herbert, "Web-based support systems with rough set analysis," in Proceedings of International Conference on Rough Sets and Intelligent Systems Paradigms (RSEISP'07), Lecture Notes in Computer Science 4585, 2007, pp. 360-370.

[13] J. T. Yao, "An introduction to web-based support systems," Journal of Intelligent Systems, vol. 17, no. 1-3, pp. 267-282, 2008.

[14] J. T. Yao and N. Azam, "Web-based medical decision support systems for three-way medical decision making with game-theoretic rough sets," Manuscript.

[15] Y. Y. Yao, "A framework for web-based research support systems," in Proceedings of the 27th Annual International Conference on Computer Software and Applications, (COMPSAC '03), 2003, pp. 601-606.

[16] Y. Y. Yao, "Decision-theoretic rough set models," in Proceedings of Second International Conference on Rough Sets and Knowledge Technology (RSKT'07), Lecture Notes in Computer Science 4481, 2007, pp. 1-12.

[17] Y. Y. Yao, "Two semantic issues in a probabilistic rough set model," Fundamenta Informaticae, vol. 108, no. 3-4, pp. 249-265, 2011.

[18] Y. Y. Yao, "An outline of a theory of three-way decisions," in Proceedings of Rough Sets and Current Trends in Computing (RSCTC'12), Lecture Notes in Computer Science 7413, 2012, pp. 1-17. 